\title{
El Maestro como formador en resiliencia para la primera infancia: Un aporte desde la escuela en la construcción de habilidades sociales
}

\author{
The Teacher as a trainer in early childhood \\ resilience: A contribution from the school in \\ the construction of social skills
}

DOI: http://dx.doi.org/10.17981/cultedusoc.11.1.2020.04

Recibido: 07/11/2019. Aceptado: 18/02/2020. Publicado: 25/02/2020

José Wilmar Pino Montoya

Universidad Católica Luis Amigó. Medellín (Colombia) jose.pinomo@amigo.edu.co

Yusty Carolina Restrepo Segura

Universidad Católica Luis Amigó. Medellín (Colombia) esp.interpsicosociales@amigo.edu.co

Luz Adriana Tobón Gómez

Centro Educativo Casita de Ilusiones. Medellín (Colombia) luztobon4155@ensa.edu.co

Leisy Magdali Arroyave Taborda

Universidad Católica Luis Amigó. Medellín (Colombia) esp.gestioneducativa@amigo.edu.co

Para citar este artículo:

Pino, J., Restrepo, Y., Tob[on, L. y Arroyave, L. (2020). El Maestro como formador en resiliencia para la primera infancia: Un aporte desde la escuela en la construcción de habilidades sociales. Cultura, Educación y Sociedad, 11(1). 55-70. DOI: http://dx.doi. org/10.17981/cultedusoc.11.1.2020.04

\section{Resumen}

El objetivo del artículo es describir el rol del docente para el desarrollo de la resiliencia y el fortalecimiento de las habilidades sociales en la primera infancia, identificando los factores de resiliencia desarrollados en estos niños. El estudio se orienta desde una metodología cualitativa para generar procesos de comprensión de la realidad, desde las voces de los actores, específicamente docentes de preescolar en relación a su mediación para desarrollar resiliencia en los estudiantes de este nivel educativo. Se conforman grupos de interés con niños de preescolar conn edades comprendidas entre 4 y 5 años. Se trabaja con instrumentos como el guión de entrevista a docentes y el sociodrama y registro de observación a estudiantes. Los resultados evidencian el rol que cumple el maestro en la implementación de estrategias pedagógicas para fortalecen la resiliencia en los niños, la cual se concibe en atención a las necesidades y etapa de desarrollo del estudiante, con el fin de favorecer su introyección. Se concluye en la importancia de un tutor resiliente en la escuela, representado especialmente en personas significativas como el docente cuyo perfil de competencias, contribuya a la mediación del proceso de acompañamiento en la formación inicial de los niños.

Palabras clave: Resiliencia educativa; habilidades sociales; primera infancia; agente educativo.

\section{Abstract}

The objective of the article is to describe the role of the teacher in developing resilience and strengthening social skills in early childhood, identifying the resilience factors developed in these children. The study is oriented from a qualitative methodology to generate processes of understanding reality, from the voices of the actors, specifically preschool teachers in relation to their mediation to develop resilience in students of this educational level. Interest groups are formed with preschool children between the ages of 4 and 5 . We work with instruments such as the script for interviewing teachers and the socio-drama and observation record of students. The results show the role played by the teacher in implementing pedagogical strategies to strengthen children's resilience, which is designed to meet the needs and stage of development of the student, in order to encourage their introjection. The conclusion is the importance of a resilient tutor at school, represented especially in significant people such as the teacher whose competence profile contributes to the mediation of the accompaniment process in the initial training of children.

Keywords: educational resilience; social skills; early childhood; educational agent 


\section{INTRODUCCIÓN}

Actualmente existe una notable preocupación por la vida de adolescentes y jóvenes, que, encuentran salidas a sus "problemas" en el consumo de drogas, alcohol, vandalismo, o peor aún; terminan en el suicidio. Estas problemáticas, aparentemente, muestran la poca capacidad juvenil de afrontar los problemas y la desmotivación de su existencia; lo que devela pocas habilidades sociales que posibiliten gestionar de forma óptima conflictos intra e interpersonales para desarrollar relaciones positivas en diferentes áreas de actuación.

El origen de este comportamiento tiene sus bases en edades más tempranas o en la etapa del ser humano denominada primera infancia (entre los 2 y 5 años), periodo donde el niño necesita, para una mayor y mejor construcción de la personalidad, el acompañamiento de personas que sirvan de de apoyo para canalizar eficientemente la resolución de sus problemas y afrontamiento adecuado de los diferentes conflictos internos y externos a los que se ve expuesto, previniendo con ello conductas conflictivas para él y el resto de la sociedad. Así, que la falta de acompañamiento, de estrategias y de procesos de socialización adecuados en los niños y niñas de entre 2 y 5 años, puede traer como consecuencia comportamientos juveniles y de los adolescentes perjudiciales para el entorno de interacción con los demás.

Esta problemática psicosocial, es posible observarla a diario en la familia y particularmente en la escuela, en cuanto que en las prácticas pedagógicas utilizadas por los maestros se hace evidente la poca tolerancia de algunos niños a la frustración frente a situaciones que desbordan sus capacidades de afrontamiento, observándose dificultades personales para el desarrollo de la empatía con el otro; poca formación en el control y comprensión de sus propias emociones y las de los demás; lo que incide en el desarrollo de la resiliencia y las relaciones sociales del niño.

Lo expuesto precisa la necesidad de generar consciencia en los profesionales de educación inicial, nivel educativo donde los niños empiezan a desarrollar características resilientes, mediado por el apoyo del adulto. Al respecto resalta el papel de los tutores en los niños a su cargo, como responsables de contribuir a la prevención y mitigación de problemáticas psicosociales futuras que afecten significativamente la vida del joven y el adolescente; se reconoce que la presencia de un adulto significativo, permite reflexionar desde la institucionalidad acerca de su quehacer desde el campo de la prevención, teniendo en cuenta que es la escuela donde se presenta la posibilidad de consolidar espacios y ambientes de construcción de resiliencia, desde la prevención y promoción del desarrollo de competencias intra e interpersonales que le permitan a los niños reconocer las situaciones de riesgo y tener la capacidad de protegerse (Muñoz y De Pedro, 2005).

Algunos estudios, desde la experiencia de formación e indagación reflexiva, destacan el papel fundamental que juega el agente educador como tutor resiliente y la importancia de contribuir desde temprana edad al desarrollo de la resiliencia y fortalecimiento de habilidades sociales, usando planteamientos evolutivos (Cyrulnik, 2002; Melillo, 2004; Peñafiel y Serrano, 2010), conocimientos actuales sobre investigaciones en resiliencia en niños de primera infancia (Reyes, 2014; Herrera, Mendoza y Guillen 2014; Pérez, 2015) y 
su aplicabilidad en el acercamiento de manera positiva del cuidador o tutor hacia el niño en el contexto familiar y escolar.

Resulta relevante preguntarse por este proceso en la primera infancia, al ser esta la etapa de las oportunidades para el reconocimiento y estímulo de interacciones permanentes del niño con el entorno familiar y social, donde la disposición del ser humano está enteramente orientada al aprendizaje y la adopción de prácticas y habilidades de afrontamiento ante la adversidad. Por otro lado, existe también la necesidad de concienciar a los maestros frente a la responsabilidad que representa como parte activa en el desarrollo integral del niño en los primeros niveles del sistema escolar.

Lo anterior, supone la orientación educativa para comprender que más allá de la resiliencia, se encuentran las habilidades sociales para contribuir al desarrollo humano del educando desde el contexto escolar; en este caso se requiere de aquella figura llamada tutor resiliente representada en el maestro que en su diario quehacer puede contribuir a la esperanza del ser, comprendida en ese ámbito escolar, como el potencial humano para trascender y transformar desde los aprendizajes significativos adquiridos en su proceso formativo.

La importancia de las habilidades sociales en la adaptación del niño a los diferentes entornos (escuela, familia, grupo de iguales entre otros), inhibe o facilitan dicha adaptación, e inciden en factores de resiliencia como la autoestima y confianza en sí mismo. Por esto se hace necesario trabajarlas desde temprana edad pues constituyen cimientos de la personalidad del niño, al no poseer estas capacidades necesarias para una adecuada adaptación a sus entornos sociales, pueden incidir en un inadecuado autoconcepto y baja autoestima, que continuará más allá de la infancia, especialmente en la adolescencia (Peñafiel y Serrano, 2010).

Así pues, es necesario evidenciar en el contexto educativo, si existen en los docentes actitudes, estrategias, acciones y alternativas para contribuir a un cambio de mentalidad en el niño para contribuir a la adaptación adecuada al contexto social, fortaleciendo su autoconcepto, autoestima y autodeterminación. En otras palabras develar, si en el ámbito educativo, en este caso en la escuela, laboran docentes que no solo forman académicamente a sus estudiantes para responder a pruebas y evaluaciones, sino docentes resilientes que eduquen integralmente a sus alumnos, un motor de desarrollo de ciertas actitudes humanas en sus educandos. En otras palabras, un agente resiliente: educador de la vida, competente como ser humano, alegre creativo y receptivo, con capacidad para escuchar y un comportamiento moral ejemplo de bienestar, un ser que garantice en un futuro inmediato el bienestar, felicidad y el ser armónico del estudiante como integrante de una sociedad.

De esta manera se pretende recobrar y recordar el espíritu y vocación del maestro brindando, con las narrativas y experiencias del docente como tutor, ofrecer una herramienta sencilla y útil para que con amor, bondad, positivismo y creatividad la utilice como medio para investigar según sus propias necesidades y construyan actividades o estrategias de intervención que contribuya a la formación de niños más fuertes y seguros y por tanto resilientes. 


\section{REVISIÓN DE LA LITERATURA}

\section{Resiliencia}

Sobre este concepto Lamas como se cita en Barrero, Riaño y Rincón (2018) manifiesta que:

El núcleo de la resiliencia es la psicología positiva, está comprendida como una postura edificadora y optimista, centrada en una perspectiva de desarrollo, en la que los seres humanos son entendidos desde sus habilidades, lo que les permitan afrontar eventos difíciles y adaptarse para sobreponerse satisfactoriamente de situaciones complejas después de haberlas experimentado (p. 122)

Para Munist et al. (1998), la resiliencia "es el resultado de un equilibrio entre factores de riesgo, factores protectores y la personalidad del ser humano. Esto último permite elaborar, en sentido positivo, factores o circunstancias de la vida que son desfavorables" (p. 14). Para Vanistendael citado en García-Vesga y Domínguez-De la Ossa (2012) se "distinguen dos componentes: la resistencia frente a la destrucción, esto es, la capacidad de proteger la propia integridad bajo presión; y, más allá de la resistencia, la capacidad para construir un conductismo vital positivo pese a circunstancias difíciles" (p. 66). Por su parte, Grotberg como se cita en Peña (2009) afirma que "la resiliencia es la capacidad humana universal para hacer frente a las adversidades de la vida, superarlas, salir de ellas fortalecido o incluso transformado positivamente por ellas" (p. 59).

Por otro lado, se entiende la resiliencia como un proceso dinámico que trae como consecuencia la adaptación positiva en contextos de adversidad; se presenta como herramienta que permite a las personas hacer frente y sobreponerse a las situaciones de desequilibrio en sus vidas ocasionado por cualquier tipo de afectación (Akl, Pilar y Aponte, 2016).

\section{Primera infancia}

El término primera infancia se halla conceptualmente imbricado a la definición de niño. Este es definido en el Programa de Apoyo para la Construcción de la Política Pública de Primera Infancia de la República de Colombia (Ley 1098, 2006), como ser social, sujeto pleno de derechos, activo y en proceso de construcción. Es concebido como un ser único, con una especificidad personal activa, biológica, psíquica, social y cultural en construcción (p. 30).

Ahora bien y para el interés particular de este artículo "se entiende por Primera Infancia el periodo de la vida, de crecimiento y desarrollo comprendido desde la gestación hasta los 7 años aproximadamente y que se caracteriza por la rapidez de los cambios que ocurren". (Jaramillo, 2007, p. 110). No obstante según Romero (2007) en el contexto colombiano "primera infancia se refiere a los niños y las niñas desde la gestación hasta los seis años (...)" (p. 44). Lo anterior se reitera en el Código de la infancia y la adolescencia (Ley 1098, art. 293, 2006) define como la "etapa del ciclo vital en la que se establecen las bases para el desarrollo cognitivo, emocional y social del ser humano. Comprende la franja poblacional que va de los cero (0) a los seis (6) años de edad". 


\section{La educación como ámbito de estudio}

Para esta investigación el concepto de educación se entiende como

Una visión del mundo y de la vida, una concepción de la mente, del conocimiento y de una forma de pensar; una concepción de futuro y una manera de satisfacer las necesidades humanas. Necesidad de vivir y estar seguro, de pertenecer, de conocerse y de crear y producir (León, 2007, p. 598).

En este sentido "la educación trata, en definitiva, de hacer a la persona mejor de lo que en un principio es, en un permanente proceso de perfeccionamiento" (Luengo, 2004, p. 37).

Para Azevedo (2013) la educación puede definirse como "la acción ejercida por las generaciones adultas sobre las generaciones jóvenes para adaptarlas a sí mismas y, en consecuencia, a su medio físico y social” (p. 84). Con la educación se pretende:

Estimular [en el ser humano] su libertad y autonomía, no segregándoles del grupo social, sino incitándolos a aprehender la propia cultura de la sociedad en la que se inserta, desarrollando la capacidad de revisar, clarificar y contextualizar los valores en beneficio propio y del grupo social en el que se desenvuelve (Liendo y Lúquez, 2007, p. 1).

Una definición más amplia e integral sobre la educación, la argumenta Campos y Restrepo (1999), quienes dicen que la educación se asume como un proceso de formación y desarrollo humano integral y, éste último, como un proceso de construcción de sentido y significado del ser humano sobre sí mismo, el mundo, la sociedad y la cultura.

Como se observa en las anteriores definiciones el concepto de educación, no alude a un concepto netamente formativo, académico, instructivo o limitado a un contexto esencial especifico, es una dimensión del desarrollo humano que se da de manera permanente, que, además, de formar y enriquecer contenidos, retoma la construcción y perfección espiritual, mental, psicológica y social de todo ser humano.

\section{El docente de la primera infancia como agente educativo}

Para argumentar el concepto de agente educativo a considerar en este artículo se retoma inicialmente a Campillo y Baldonero (2013), para quienes:

Los agentes educativos son todas aquellas personas que trabajan en Educación Inicial (y). Su participación es determinante en la construcción de un servicio que reconoce la condición vulnerable del ser humano (...) que concentra su acción educadora en el proceso de desarrollo y transformación de sus capacidades intelectuales, emocionales y físicas a través de un conjunto de experiencias de aprendizaje (p. 7).

Para el Ministerio de Educación Nacional-MEN (2005) un agente educativo "es un formador de seres humanos que pasa más tiempo con los niños y jóvenes, se hace evidente la importancia social de su trabajo y las diversas responsabilidades de su profesión" (p. 4). Entre estas responsabilidades "el maestro, según lo consideraban los griegos, era quien formaba el carácter del discípulo y velaba por el desarrollo de su integridad moral, orientada a la formación del alma y al cultivo respetuoso de los valores éticos y patrióticos" (De Cleves, Velásquez y Calle, 2004, p. 267). 
De lo anterior se deduce que para que el maestro cumpla adecuadamente su rol no basta con su formación científica y académica, debe también tener competencias afectivas y personales, pues (...) "una de sus responsabilidades es cuidar el desarrollo de su estudiantado, de sus experiencias de aprendizaje, autoestima, sensibilidad, así como de su creatividad, de manera que puedan ser actores con posibilidad de participación en proyectos de bien social" (Astudillo y Chávez, 2015, p. 163).

\section{Habilidades sociales}

Autores como Monjas citado en García (2011) refiere a las habilidades sociales como: "las conductas o destrezas sociales específicas requeridas para ejecutar competentemente una tarea de índole interpersonal. Es decir, las habilidades sociales son un conjunto de comportamientos interpersonales complejos que se ponen en juego en la interacción con otras personas" (p. 5). Para Ballester y Gil como se cita en Contini y Betina (2009) "la competencia social proporciona sentimientos de autoeficacia que constituye un ingrediente fundamental de la autoestima. Ser reconocido y aceptado por otro produce un impacto favorable en la autoestima, y ello refuerza la satisfacción vital" (p. 4). Por su parte, Crespo y Prieto como se cita en Contini y Betina (2009) dicen que las habilidades sociales son un medio excepcional de protección y promoción de la salud ya que los comportamientos sociales positivos favorecen la adaptación, la aceptación de los otros, los refuerzos positivos y el bienestar del sujeto" (p. 57). Contini y Betina (2009), hacen una relación entre los niños y las habilidades sociales, advierten que:

En niños y adolescentes, la temática de las habilidades sociales es relevante, no sólo por su dimensión relacional, sino por su influencia a otras áreas vitales tales como la escolar, la familiar, entre otras. Está comprobado que aquellos niños y/o adolescentes que muestran dificultades en relacionarse o en la aceptación por sus compañeros del aula, tienden a presentar problemas a largo plazo vinculados con la deserción escolar, los comportamientos violentos y las perturbaciones psicopatológicas en la vida adulta (p. 161)

Cabe entonces decir que las habilidades sociales "inciden en la autoestima, en la adopción de roles, en la autorregulación del comportamiento y en el rendimiento académico, entre otros aspectos tanto en la infancia como en la vida adulta" (Betina, s.f., p. 233). De allí que un sujeto con capacidad de relacionarse con los otros o con habilidades sociales, es un ser que se fortalece a sí mismo, con capacidad de ejercer lo que es y para lo que se formado adecuadamente, autónomo y competente en el rol para lo que se ha educado. Es lo que denominan autores como Gutiérrez, Carrera, Marín, Narváez y Pérez (2006), la capacidad que tienen los actores de interactuar y establecer redes relacionales como base para el desarrollo humano y social.

\section{Metodología}

Se trata de una investigación cualitativa que permite describir desde la vivencia cotidiana, la comprensión de los maestros en relación a su función en el desarrollo de la resiliencia de sus estudiantes; también se reconocen características de los niños en primera infancia que trazan el camino hacia su construcción como personas resilientes (Sandín, 2003). 
Se trabaja la revisión de literatura sobre la resiliencia en la primera infancia, factores y característica de un tutor resiliente, y conocimientos acerca de las habilidades sociales. El escenario de intervención es el grado Jardín de la Institución Casita de Ilusiones ubicada en el barrio la colina en Guayabal del municipio de Medellín; la sección está integrada por 18 niños con edades comprendidas entre 4 y 5 años; para la conformación del grupo de interés se seleccionan 6 niños, cuyo comportamiento exhiba mayor o menor sentido resiliente en sus actividades rutinarias de socialización y adaptación.

Como técnicas para la recolección de los datos, se utilizan la entrevista semiestructurada a tres maestras y a los 6 niños, el sociodrama y la observación intencionada, a través de instrumentos como el guion de entrevista y el registro de observación. El análisis de la información desde la inferencia permite la comprensión teórica de los constructos identificados.

\section{RESULTADOS Y DISCUSIÓN}

Después de haber esbozado los referentes teóricos que guían la triangulación de los datos y el análisis de la información, el siguiente apartado contempla los resultados encontrados, aludiendo, en primer lugar al análisis de la revisión de literatura científica existente sobre esta temática; en un segundo momento a los conocimientos y características que tienen los docentes para formar y desarrollar en la primera infancia la capacidad resiliente; y en tercer lugar los hallazgos que describen a los niños y niñas como resilientes ante situaciones hipotéticas que podrían afrontar.

Aproximación a la revisión de literatura científica sobre la resiliencia en la primera infancia

Al hablar de resiliencia, es posible identificar que, aunque es un tema reciente, son muchos los investigadores que se han orientado hacia este campo, aspecto que revela el interés no solo por mencionar el término sino por indagar sobre su repercusión y su proceso de adquisición a partir de la primera infancia.

Es así como Reyes (2014), en su estudio factores resilientes de un grupo de niños de 4 a 6 años con anemia aplástica, revela como ante la adversidad representada en enfermedades graves, los niños a pesar de su corta edad, son capaces de mostrar autoestima, introspección, iniciativa e independencia para hacerle frente a su proceso de recuperación, delegando una función primordial a los adultos significativos, quienes asumen el rol de tutores resilientes, al ser los responsables de enseñar y transmitir a los niños las formas, estrategias y aprendizajes para hacerle frente a los problemas, lo que resulta en el acompañamiento asertivo para adquirir la resiliencia.

Por su parte, Pérez (2015), en su artículo titulado resiliencia en la infancia, presenta un modelo de prevención y promoción basado en el desarrollo de potencialidades y recursos de los niños, orientado desde la importancia de la presencia del adulto para generar interacciones sanas en los diferentes entornos de socialización del niño; el adulto cumple un rol protagonista como agente significativo que desarrolla y promueve el desarrollo pleno de las habilidades sociales y la capacidad resiliente de los niños, rescatando los aspectos positivos de la vida y permitiendo el verdadero desarrollo humano de la infancia en aras de reducir el riesgo y las fuentes de estrés en la vida del niño. 
De igual manera, Herrera, Mendoza y Guillen (2014), en su investigación resiliencia: proceso de afrontamiento de la dimensión personal social en la educación inicial, mencionan que la intervención del adulto es primordial para el desarrollo de procesos resilientes en niños de primera infancia, y que es posible, a partir de estrategias pedagógicas, la promoción de capacidades de afrontamiento ante la adversidad, favoreciendo la expresión de emociones y sentimientos ante las diferentes situaciones, y buscando fortalecer los factores interpersonales e intrapersonales que permitan afrontar de una manera asertiva el estrés y la frustración, es allí donde debe estar el adulto ofreciendo afecto, cariño, buen trato y acompañamiento para la enseñanza de estas capacidades.

Por último, Gallego y Cortines (2014), en el artículo titulado la resiliencia como enfoque pedagógico: Hacia una mirada transformadora del hacer docente, destacan que la resiliencia de los niños depende del trabajo mancomunado de la familia, docente y la comunidad, como actores corresponsables de los procesos de enseñanza-aprendizaje; relevando el rol del maestro como ente socializador en el proceso educativo, quien desde su propia vocación orienta el sano desarrollo de los estudiantes. De modo que, desde su visión docente, tienen la posibilidad de hacer lectura tanto de factores de riesgo que afectan sus estudiantes, como la identificación de factores protectores del entorno que contribuyan al desarrollo de la capacidad para superar problemas.

Definitivamente, cuando de la infancia se trata, es necesario que la responsabilidad de su desarrollo, aprendizaje y crecimiento personal, sea asumido por adultos gestores de cambios y aprendizajes significativos, capaces de construir y brindar al niño ambientes de aprendizaje saludables, con capacidad de dar y recibir afecto, y sobre todo con la convicción de asumir un rol protagonista para la formación de seres humanos en desarrollo, que requieren del mayor compromiso posible del maestro y las familias para garantizar el desarrollo pleno de capacidades y potencialidades humanas.

\section{Maestros de primera infancia constructores de resiliencia}

La resiliencia en la primera infancia, no es un proceso consciente, concibe al adulto que acompaña la formación de los niños, como responsable de generar estrategias donde el infante desarrolle esta habilidad, pero a su vez supone que estos adultos cuenten con unas características personales para mediar este proceso en el niño.

Al indagar en los docentes del contexto investigado sobre este concepto, son evidentes las debilidades en varios aspectos, pues, aun conocedoras de sus profesión, así como de sus capacidades para intervenir en el desarrollo de habilidades en los niños, parecen no estar muy conscientes de sus competencias para intervenir propositivamente en el desarrollo de un perfil resiliente en los niños; se infiere de aseveraciones como: "la resiliencia para mí, son los niños que muestran cierta apatía por algo, no desean hacer nada" (M3). En correspondencia con este hallazgo, la Fundación Bernard Van Leer (2002), sustenta que los programas de educación infantil bajo un enfoque de resiliencia, deben tener en sus compromisos la responsabilidad de promover a través de diferentes estrategias pedagógicas, especialmente el juego, las diferentes características para potenciar en niños resilientes, autonomía, autoestima, creatividad y humor (p. 33). 
No obstante, existen opiniones que demuestran cierto conocimiento al respecto, una de las entrevistadas afirma lo siguiente "la resiliencia es cuando alguien ha sufrido mucho y a pesar de eso, ya está bien" (M2). Según esta apreciación, es usual encontrar que cuando se habla de formación o educación, casi siempre esta responsabilidad tiende a recaer en el sistema educativo, específicamente en los docentes responsables de la educación infantil. Sin embargo, la formación en resiliencia demanda, especialmente, un trabajo cooperativo, lo que implica más allá del trabajo de los maestros en sus aulas, una participación activa de las familias como entes corresponsables no solo de acompañar el proceso formativo sino también de promoverlo y potenciarlo desde el hogar. Ello implica la cualificación a docentes y familias para permitir mejorar las condiciones educativas de los niños y los ambientes donde adquieren sus conocimientos como potenciadores del desarrollo humano y personal (Acevedo y Restrepo, 2012, p. 313).

Ante los niños resilientes, los informante clave identifican características básicas, sustentando sus respuestas en comportamientos o expresión de emociones, pero no en características personales, o en capacidades para hacer frente a las adversidades. Se evidencia en los aportes de una informante: "un niño no resiliente es rebelde, y apático y el que sí lo es, es alegre, ayuda y juega bien" (M1). Se infiere que las habilidades sociales se encuentran asociadas al desarrollo de la capacidad resiliente, al potenciador las relaciones interpersonales, el trabajo cooperativo, la capacidad de comprender las propias emociones y las de los demás, y la capacidad altruista de brindar ayuda a quien lo necesita. Ello se corresponde con lo expuesto por Peñafiel y Serrano (2010), cuando señala que las habilidades sociales se encuentran estrechamente relacionadas con el tipo de conductas esperadas en las diferentes interacciones del niño, y terminan influyendo en su adaptación a los diferentes entornos. La Informante clave 2 confirma lo anterior: "un niño resiliente está feliz, es cariñoso, ayuda, juega, comparte y respeta, el que no es resiliente, llora, hace pataletas. No le gusta trabajar, pelea" (M2)

Al considerar las reacciones de niños ante situaciones de adversidad, estrés o descontento, las maestras fundamentan sus respuestas en comportamientos y acciones relacionadas con la edad. Al respecto la percepción de un informante clave: "las reacciones de un niño resiliente ante situaciones de angustia y frustración, es que no llora mucho, son puntuales, busca ayuda, viene donde mí” (M1). A este respecto, Pereira (2010) señala que:

La resiliencia se construye cuando nos relacionamos, es decir, ningún niño tiene la posibilidad de volverse resiliente por sí solo, necesita a los otros, quienes brinden cuidado, protección, comodidad y respaldo ante la adversidad, que lo respete, le enseñe a valorarse y a confiar en sus propias capacidades, y además le enseñé a respetar a los demás. (p. 12).

Si bien se evidencia confusión frente al término en una de las informantes, quien responde de manera equívoca ante el significado del concepto resiliencia, se nota claridad sobre aquello que limita en el ser humano la posibilidad de ser resiliente, ello interfiere frente a su rol para acompañar y apoyar el desarrollo de capacidades y habilidades sociales, donde los niños adopten posturas y estrategias para hacerle frente a los obstáculos. Lo anterior se refleja en las verbalizaciones: "los niños resilientes no pueden hacer nada de lo que desean, lo demuestran a través de pataletas" (M3). Complementariamente las entrevistadas aluden: "hay niños que son resilientes porque viven en condiciones malucas" (M1). "Los niños no desarrollan resiliencia porque carecen de amor y acompañamiento" (M2). 
Las percepciones expuestas corresponden a los informantes clave; indican que el desarrollo de estas habilidades y capacidades resilientes, necesitan de la presencia de adultos guías y formadores de niños resilientes, conforme a lo argumentado por Henderson y Milstein (2005), entre más pequeño es el niño, su necesidad de apoyo de un adulto significativo es mayor; es posible caracterizar el funcionamiento del adulto como tutor resiliente; debe cumplir con características específicas para desempeñar un rol que inspire confianza al niño, donde reconozca los límites y proteja del riesgo y del peligro, y fundamentalmente ha de ser una persona cuya conducta ejemplifica las formas de proceder ante las diferentes situaciones de la vida.

Los informantes clave no parecen ser muy conscientes del rol fundamental que representan y cumplen en la formación humana de sus estudiantes, pues es notable la dificultad para reconocerse como agente generador de cambio y constructor de seres humanos resilientes. No obstante, es posible inferir que los docentes de primera infancia, necesariamente influyen en los aprendizajes a introyectar en los niños en relación a la forma de resolver problemas, hacer frente a la vida o recuperarse ante las adversidades.

\section{Relación entre construcción de resiliencia en la primera infancia y adquisición de habilidades sociales}

Con respecto a esta categoría, se evidencia que los niños con mayor capacidad de resiliencia, muestran mejores habilidades sociales y de afrontamiento frente a las situaciones difíciles, de estrés o de conflicto interno, basando sus respuestas en afirmaciones, verbalizaciones positivas y actitudes asertivas para la resolución de conflictos, tal como se desprende de la siguiente afirmación: "cuando tengo un problema lo vuelvo a hacer, cuando tengo miedo corro y los salvo, yo no necesito ayuda porque puedo solo esquivarlo como Ironman" (Alumno 6)

Aquí, la resiliencia puede entenderse como aquella adaptación positiva del ser humano para hacer frente a los contextos adversos. En los primeros años de vida está cobra relevancia debido a que las intervenciones de los adultos significativos en estas etapas, permiten prevenir y reducir los riesgos psicosociales, además fomenta las competencias, aumentan los recursos internos y se construyen los cimientos para un desarrollo integral (Masten y Gewirtz, 2008, p. 1).

En cuanto a los niños con menor capacidad resiliente adoptan una actitud negativa frente a las situaciones adversas; responden con verbalizaciones de enojo, confusión, dificultades para la toma de decisiones, y finalmente una actitud de huida o evasión de las situaciones de adversidad. El texto siguiente es ilustrativo de lo señalado:

Cuando algo me sale mal vómito y eructo, cuando alguien hace chiste de lo malo porque me pegan, porque me siento mal (o) cuando tengo un problema lloro, me voy, en una situación peligrosa me voy, cuando necesito ayuda lloro (Alumno 2).

Lo expuesto sugiere, que algunos niños lograrán adquirir y construir su capacidad resiliente a partir de la exposición al medio escolar, social y familiar, mientras que otros requerirían de apoyos significativos y de la presencia de adultos capaces y estables para obtener mayor seguridad y acompañamiento oportuno en el proceso. El desarrollo mental del niño depende de las experiencias y vivencias ofrecidas por el adulto, así como de la 
riqueza adaptativa en situaciones que puedan significar adversidad, aversión o dificultad, pero siempre con la guía de aquellos tutores o guías este proceso.

Al respecto se puede tomar el comentario de Henderson y Milstein (2005), al afirmar que las escuelas son espacios fundamentales donde los niños aprender a sobreponerse a la adversidad y adquieren competencias sociales para la búsqueda de relaciones humanas de reciprocidad; señalan siete pasos para llevar a cabo el proceso de resiliencia: mitigar el riesgo y enriquecer los vínculos; fijar límites firmes; enseñar habilidades para la vida; construir la resiliencia; brindar afecto y apoyo; establecer expectativas elevadas y brindar oportunidades de participación significativa.

En el mismo sentido, es posible revelar el altruismo en los niños más resilientes, como esa capacidad para ponerse en los zapatos del otro, comprender sus propias emociones y las de los demás, además de brindar ayuda cuando el otro lo requiere, y pedirla cuando no cuenta con los recursos necesarios para afrontarlo. En este momento se puede resaltar nuevamente al alumno 2: "en mi casa ayudo dando de comer a los pájaros, en la escuela ayudo con los niños, cuando alguien necesita ayuda lo rescato."

En este mismo sentido, Rodríguez (2002) reconoce que la competencia social permite formar niños resilientes; se observa en las respuestas asertivas frente al contacto con otros seres humanos, mostrando reacciones positivas, flexibles y que se adaptan fácilmente a los diferentes entornos, por medio de una comunicación desde la empatía y el afecto reflejados como comportamientos prosociales.

Por el contrario, los niños con menor capacidad de resiliencia, evidencian conductas egoístas, de burla o de pasividad frente a la necesidad de ayuda del otro, además se muestran confundidos cuando afrontan una situación difícil verbalizando no saber qué hacer o con reacción de llanto. Tal como: "cuando algo sale mal yo me rio de los amigos, y si hacen chistes de lo malo es porque son groseros" (Alumno 3).

Ante las anteriores circunstancias y como lo afirma Pereira (2010) es necesario concebir la resiliencia como proceso dinámico, a lo largo del tiempo, y sustentado en la posibilidad de interactuar con otras personas y el entorno, entre la familia y el medio social; ante la adversidad, los sistemas en su amplitud, familia, escuela, organizaciones, grupos sociales y adultos significativos son quienes logran proveer al niño de protección facilitando la resiliencia infantil.

También se constata, que los niños más resilientes, poseen recursos para comprender que ante la adversidad es posible encontrar soluciones asertivamente, destacando sus capacidades relacionales con el otro, el mundo y las circunstancias de manera positiva a pesar de la situación adversa, y mostrando competencias para buscar ayuda de ser necesario en los momentos difíciles para tomar decisiones y afrontar el estrés. “(...) cuando no puedo solucionar algo, mi abuelo y mi abuela me ayudan” (Alumno 6). Este mismo comportamiento lo evidencia el testimonio del alumno 4: "cuando tengo un problema, le pido ayuda a mi abuela, cuando hay peligro le digo a mi abuela, yo no tengo miedo". Por el contrario, los niños menos resilientes, en este aspecto no logran comprender que la adversidad puede traer ventajas o convertirse en situaciones favorecedoras, sus capacidades de relacionamiento con el otro se tornan torpes u obstaculizadas al sentirse rechazados por sus pares y carecen de autonomía para hacer frente a los problemas incluso ante aquellos simples de solución. “(..) Cuando las personas hacen chistes de lo malo, hablan puras mentiras” (Alumno 1). 
Es este tipo de casos donde el maestro como tutor resiliente, según Cyrulnik (2005), debe contar con unas características esenciales que le permitan cumplir con este rol en la infancia, como capacidad de relacionarse sanamente, la creatividad de resignificar el dolor, el humor para dar sentido a las adversidades, la autonomía para fijar y mantener los límites entre su integridad personal y los problemas, iniciativa de exigirse a sí mismo y ponerse a prueba, la introspección que le permite preguntarse y darse respuestas así mismo, el sentido de la moral para comprometerse con los valores personales, la confianza en sí mismo como factor protector que conlleve a superar cualquier evento frustrante y la capacidad de encontrar sentido a lo acontecido.

En este sentido, se hace necesario comprender la resiliencia en la infancia, como un camino por recorrer, porque implica el acompañamiento al niño en su proceso evolutivo no solo a nivel físico, sino también social, emocional y mental, teniendo en cuenta la concientización del adulto del tipo de acompañamiento, de sus características como tutor y de sus capacidades para orientar lo necesario en momentos adversos como base de un aprendizaje significativo y duradero.

El docente puede promover la resiliencia en sus niños como agente de cambio; propicia más allá del aprendizaje académico, la formación humana, siendo un ejemplo de vida a partir de su capacidad para desarrollarse, autodirigirse y encaminar sus acciones educativas y de formación integral a sus educandos (Aguaded y Almeida, 2016, p. 170). Conviene subrayar en este aspecto, que es la dimensión personal de educador, lo que permitirá en el estudiante reflejar su propia resiliencia, a partir de sus intervenciones educativas para favorecer este proceso; así mismo conlleva la necesidad de garantizar la cualificación necesaria para el fomento del aprendizaje personal, afectivo y emocional, con el consecuente desarrollo humano y social.

\section{Conclusiones}

Los resultados de investigación, permite evidenciar que la capacidad del niño resiliente se refleja en aquellos comportamientos, pensamiento, verbalizaciones y decisiones, para tomar frente a las condiciones adversas; es visible en niños con una mejor capacidad resiliente, expresada en autonomía en la toma de decisiones, iniciativa para resolver problemas y seguridad frente a sus actos frente a la realidad.

Por otra parte se evidencia que aunque en la educación se ha venido innovando frente a los medios, estrategias y didácticas para promover el aprendizaje, sigue habiendo un notable desconocimiento del papel a cumplir por la inteligencia social y emocional para la formación integral de los seres humanos desde su niñez.

Muestra de ello es la formación impartida en las instituciones educativas, medida desde el logro de objetivos académicos más que en la formación personal y humana; si bien existe una normatividad para garantizar los derechos fundamentales del niño, entre ellos el desarrollo pleno de su personalidad y la garantía de su protección y bienestar integral, parece no estar contemplado, el desarrollo humano no como un factor inherente a la sociedad, sino como un asunto de construcción colectiva.

El proceso de construcción colectiva involucra los diferentes contextos y escenarios de interacción para los niños; en este ámbito se comprende que la familia, las instituciones 
educativas representadas en el maestro y la sociedad, deben garantizar los entornos adecuados para propiciar el desarrollo de competencias y habilidades de adaptación y desarrollo.

El estudio evidencia que las familias y docentes, constituyen el sistema de apoyo de los niños de primera infancia, pues son sus principales cuidadores y las personas con quien comparten más tiempo; el reto se orienta a la comprensión de los actores de procesos formativos, donde no solo se involucren la adquisición de conocimientos académicos, sino la atención al desarrollo humano y personal de los niños en los diferentes entornos educativos. Este asunto requiere del trabajo mancomunado para concientizar y lograr la corresponsabilidad con el desarrollo personal, social y emocional de los niños para viabilizar su adaptación a las diferentes circunstancias de la vida.

Para desarrollar resiliencia en infantes, necesariamente debe existir el compromiso conjunto de las personas que rodean los niños en su vida diaria, comprendiendo la familia como ente principal socializador y educativo, así como la escuela representada por el docente y la comunidad como protectora y formadora de la infancia.

El maestro tiene un rol significativo en la formación de los niños, su presencia es fundamental, especialmente cuando las condiciones sociales y familiares no favorecen el proceso de desarrollo humano de los niños, es allí donde puede adoptar el rol de tutor resiliente, a partir de características específicas que lo hacen apto y un modelo a seguir en el contexto escolar. También en este proceso es importante la responsabilidad formativa no solo de los maestros sino también de la familia, y el estado. Resalta la necesidad de la presencia del Estado para ofrecer a maestros y familias la cualificación pertinente que los lleve a comprender la importancia de la vida interior, social y emocional de los niños.

Todo lo expuesto, implica que el maestro reciba una formación profesional desde lo humano, donde necesariamente se trabaje la autoestima, la creatividad, la iniciativa y el sentido del humor, encaminado hacia el desarrollo de la empatía humana, las habilidades sociales y la actitud positiva ante la vida, para que sin importar las circunstancias y los contextos donde esté inmerso, mantenga actitudes resilientes desde la asertividad, para contribuir a mejorar la calidad de vida de los niños y marcando sus vidas desde una postura positiva.

\section{REFERENCIAS}

Acevedo, V. E. y Restrepo, L. (2012). De profesores, familias y estudiantes: fortalecimiento de la resiliencia en la escuela. Revista Latinoamericana de Ciencias Sociales, Niñez y Juventud, 10(1), 301-319. Recuperado de http://www.umanizales.edu.co/publicaciones/campos/cinde/index.html

Aguaded, M. y Almeida, N. (2016). La resiliencia del docente como factor crucial para superar las adversidades en una sociedad de cambios. Tendencias pedagógicas, (28), 167-180. Recuperado de https://revistas.uam.es/tendenciaspedagogicas/article/viewFile/3831/5211

Akl, P., Pilar, E. y Aponte, F. (2016). Estrategias de afrontamiento en mujeres víctimas de violencia intrafamiliar. Cultura Educación y Sociedad, 7(2), 105-121. Disponible en https://revistascientificas.cuc.edu.co/culturaeducacionysociedad/article/view/1105/ pdf_238 
Astudillo, M. y Chávez, F. (2015). Agentes e instituciones de la educación: una reflexión desde las desigualdades sociales. Ciencia Ergo Sum, 22(2), 161-166.

Azevedo, F. (2013). Sociología de la educación. Introducción al estudio de los fenómenos pedagógicos y de sus relaciones con los demás fenómenos sociales. México, D.F.: FCE. Recuperado de https://www.ssoar.info/ssoar/bitstream/handle/document/45829/ ssoar-cienciaergo-2015-2-astudillo_torres_et_al4-Agentes_e_instituciones_de_ la.pdf?sequence $=1 \&$ isAllowed $=\mathrm{y}$

Barrero, A. M., Riaño, K. T. y Rincón, L. P. (2018). Construyendo el concepto de la resilencia: una revisión de la literatura. Poiésis, (35), 121-127. https://doi. org/10.21501/16920945.2966

Betina, A. (s.f.). Las habilidades sociales como recursos para el desarrollo de fortalezas en la infancia. Psicodebate, (10), 231-248.

Campo, R. y Restrepo, M. (1999). Formación Integral: Modalidad de educación posibilitadora. Bogotá, D.F.: Pontificia Universidad Javeriana.

Campillo, M. y Baldonero, M. (2013). Ser agente educativo. Cuauhtémoc: Secretaría de Educación Pública.

Contini, N. y Betina, A. (2009). Las habilidades sociales en niños preescolares en contextos de pobreza. Ciencias Psicológicas, 3(1), 57-66. Disponible en http://www.scielo. edu.uy/scielo.php?pid=S1688-42212009000100006\&script=sci_arttext

Cyrulnik, B. (2005). El amor que nos cura. Barcelona: Gedisa.

Cyrulnik, B. (2002). Los patitos feos. Barcelona: Gedisa. Recuperado de https://www.raco. cat/index.php/EducacioSocial/article/viewFile/169585/381188

De Cleves, R. Velásquez, B. y Calle, M. (2004). El maestro como formador y cultor de la vida. Tabula Rasa, 2(1), 263-281. Recuperado de http://www.revistatabularasa.org/ numero-2/calle.pdf

Fundación Bernard Van Leer. (2002). Resiliencia en programas de desarrollo infantil temprano: estudio de revisión en cuatro programas de América Latina. La Haya: Autores.

Gallego, I. y Cortines, L. (2006). La resiliencia como enfoque pedagógico: hacia una mirada transformadora del hacer docente. [Tesis de pregrado]. Universidad de Antioquia, Medellín, Colombia. Recuperado de http://ayura.udea.edu.co:8080/jspui/bitstream/123456789/1630/1/CA0106.pdf

García-Vesga, M. C. y Domínguez-De la Ossa, E. (2013). Desarrollo teórico de la Resiliencia y su aplicación en situaciones adversas: Una revisión analítica. Revista Latinoamericana de Ciencias Sociales, Niñez y Juventud, 11(1), 63-77. Disponible en https:// www.redalyc.org/articulo.oa?id=773/77325885001

García, M. (2011). Habilidades sociales en niños y niñas con discapacidad intelectual. [Online]. Recuperado de http://www.eduinnova.es/mo4nografias2011/ene2011/habilidades.pdf.

Gutiérrez, C., Carrera, M., Marín, F., Narváez, M. y Pérez, C. (2006). Integración de redes académicas para la gestión del desarrollo endógeno regional. Multiciencias, 6(3), 257-263. Disponible en https://www.redalyc.org/pdf/904/90460308.pdf

Henderson, N. y Milstein, M. (2005). Resiliencia en la escuela. México, D.F.: Paidós. 
Herrera, D., Mendoza, G. y Guillen, Y. (2014). Resiliencia: proceso de afrontamiento de la dimensión personal social en la educación inicial. [Trabajo final de grado]. Corporación Universitaria Minuto de Dios, Bogotá, D.C., Colombia. Recuperado de https:// repository.uniminuto.edu/bitstream/handle/10656/2925/TPED_GuillenPatinoLuisa_2014.pdf?sequence $=1 \&$ isAllowed $=y$

Jaramillo, L. (2007). Concepciones de infancia. Zona Próxima, 8, 108-123. Recuperado de http://rcientificas.uninorte.edu.co/index.php/zona/article/viewfile/1687/1096

León, A. (2007). Qué es la educación. Educere, 11(39), 595-604. Disponible en https:// www.redalyc.org/pdf/356/35603903.pdf

Luengo, L. (2004). La educación como objeto de conocimiento. El concepto de educación. En, M. Pozo, J. Álvarez, J. Luengo y E. Otero, Teorías e instituciones contemporáneas de educación (30-47). Madrid: Biblioteca Nueva.

Liendo, Z. y Lúquez, P. (2007). Eje Transversal Valores: Epistemología y Fundamentos Curriculares en la Práctica Pedagógica de Educación Básica. Laurus, 13(25), 82-113. Disponible en https://www.redalyc.org/comocitar.oa?id=76111479005

Masten, A. y Gewirtz, A. (2008). Resiliencia en el desarrollo. La importancia de la primera infancia. Enciclopedia sobre el Desarrollo de la Primera Infancia. [Online]. Recuperado de http://www.enciclopedia-infantes.com/resiliencia/segun-los-expertos/ resiliencia-en-el-desarrollo-la-importancia-de-la-primera-infancia

Melillo, A. (2004). Resiliencia. Revista Psicoanálisis: ayer y hoy, (1). Recuperado de https:// www.elpsicoanalisis.org.ar/old/impnumero1/resiliencia1-doc.htm

Muñoz, V. y De Pedro, F. (2005). Educar para la resiliencia. Un cambio de mirada en la prevención de situaciones de riesgo social. Revista Complutense de Educación, 16(1), 107-124. Recuperado de https://revistas.ucm.es/index.php/RCED/article/view/RCED0505120107A

Munist, M., Santos, H., Kotliarenco, M. A., Suárez, E. N., Infante, F. y Grotberg, E. (1998). Manual de identificación y promoción de la resiliencia en niños y adolescentes. Washington, D.C.: Fundación W.K., Kellogg Autoridad Sueca para el Desarrollo Internacional (ASDI).

Peña, N. E. (2009). Fuentes de resiliencia en estudiantes de Lima y Arequipa. Liberabit. Revista Peruana de Psicología, 15(1), 59-64. Disponible en https://www.redalyc.org/ articulo.oa?id=686/68611923007

Peñafiel, E. y Serrano, C. (2010). Habilidades Sociales. Madrid: Editex. Recuperado de https://docplayer.es/28278596-Habilidades-sociales-eva-penafiel-pedrosa-cristina-serrano-garcia.html

Pereira, R. (2010). Trabajando con los recursos de la familia: factores de resiliencia familiar. Revista Sistemas Familiares, 26(1), 96-115. Recuperado de http://congresonacionaldepsicoterapia.es/wp-content/uploads/2017/04/Trabajando-cn-los-recursos-de-lafam.-Factores-de-Resiliencia-Fam.-R.-Pereira.pdf

Pérez, L. (2015). Promoción de Resiliencia En la Infancia. [Trabajo final de grado]. Universidad de la República, Asunción, Uruguay. Recuperado de https:/sifp.psico.edu.uy/ sites/default/files/Trabajos\%20finales/\%20Archivos/tfg_-_lucia_perez.pdf 
República de Colombia. Congreso de la República de Colombia (8 de noviembre de 2006). Código de la Infancia y la Adolescencia. [Ley 1098]. Diario Oficial No 46.446. Recuperado de https://www.icbf.gov.co/cargues/avance/docs/ley_1098_2006.htm

República de Colombia. MEN. (2005). Enseñar para la vida, Altablero, (34), 1-20. Recuperado de https://www.mineducacion.gov.co/1621/propertyvalue-31232.html

Romero, T. (2007). Colombia por la primera infancia: ¿utopía o realidad? Reflexiones sobre la política pública para la población menor de seis años. Revista Colombiana de Educación, (53), 40-57. Disponible en https://www.redalyc.org/articulo. oa?id=4136/413635247003

Reyes, W. (2014). Factores resilientes de un grupo de niños de 4 a 6 años con anemia aplástica. [Tesis de grado]. Universidad Rafael Landíva, Asunción, Guatemala. Recuperado de http://biblio3.url.edu.gt/Tesario/2014/05/42/Reyes-Wenndy.pdf

Rodríguez, A. (2002). Resiliencia. Psicopedagogía, 26(80), 291-302. Recuperado de http:// pepsic.bvsalud.org/pdf/psicoped/v26n80/v26n80a14.pdf

Sandín, E. (2003). Investigación Cualitativa en Educación. Fundamentos y Tradiciones. Revista de pedagogía, 26(77), 48-58. Recuperado de http://www.scielo.org.ve/scielo. php?script=sci_arttext\&pid=S0798-97922005000300007 